\title{
Visualization of Crystal Orientation and the Granulate Sub-Grain Size in Lithium Ion Batteries by Means of EBSD and Correlative Microscopy
}

\author{
Daria Zeibig ${ }^{1}$, Dr. Timo Bernthaler ${ }^{1}$, Prof. Dr. Gerhard Schneider ${ }^{1}$, Stefanie Freitag ${ }^{2}$ \\ 1. Materials Research Institute at Aalen University, Aalen, Germany \\ 2. ZEISS Microscopy GmbH, Business Sector Materials Science, Munich, Germany
}

Secondary or rechargeable batteries are of interest for low power applications, for example in consumer electronics but also for research fields that are driven by the growing need for high power like electric transportation systems. In order to understand the lithium storage and release potential in a mixed lithium manganese oxide and lithium manganese cobalt battery cathode, the crystal orientation and granulate sub-grain size was investigated in this study.

A classical preparation method in this context is a combination of embedding in resin, cutting, grinding and mechanical polishing. But due to the high sensitivity of lithium ion battery materials to air and moisture the mostly water assisted processes of grinding and polishing are predestinated to cause problems when applied to LIB materials. A promising, water free and oxygen free alternative to obtain the desired surface quality for LIB materials is the preparations of cross sections by means of a focused ion beam (FIB) implemented into an electron microscope. However, the removal of material by FIB is limited to very small amounts of material and in order to perform crystallographic imaging, called electron backscattered diffraction (EBSD) measurements, free standing, exposed surfaces are needed.

To prepare the desired cross sections of the battery active material meeting these requirements, much higher removal of specimen material is needed. To realize this, a laser system can be applied. The laser pretreatment of the cathode foil sample allows to remove even large parts of the sample to yield free standing, exposed cross section surfaces of the cathode foils from quasi everywhere across the foil.

The cathode material investigated was extracted from commercial 18650 type lithium-ion-batteries featuring composite cathode active material composed of equal amounts of spinel-type lithium manganese oxide LiMn2O4 (LMO) and lithium manganese cobalt nickel oxide (1:1:1) LiMn1/3Co1/3Ni1/3O2 (NMC). The anode active material was based on graphite.

The cells were discharged and afterwards opened in an argon filled glovebox to ensure protective atmosphere especially absence of oxygen and moisture. Subsequently, the jelly roll was unrolled; the electrode foils were separated from the separator, washed with dimethyl-carbonate (DMC), and dried in an oven at $70^{\circ}$. Small pieces of the cathode foil were cut by scissors and mounted on a SEM stub in a way to jut out beyond the edge of the stub. The piece of cathode foil selected was precut by a laser system (Trumpf Trumark 6000). Subsequently the projection was fine-cut by a FIB-milling procedure involving several preparation steps, which were characterized by successively decreasing FIB currents to yield a smooth surface with only little residues of so called curtaining, a kind of waviness of the cross section surface introduced by the FIB. A smooth surface without artefacts is crucial for reliable EBSD measurements since EBSD is a very surface-sensitive method sampling only the topmost $40 \mathrm{~nm}$ of the specimen [1]. The revealed FIB cross-section showed the NMC as well as the LMO particles. Both particle-types are very compact arranged with relatively low pore space. The LMO particles in this cross-section do mainly occur at the surface of the section. The NMC particles do exhibit a sub-grain 
structure. The EBSD measurements were performed by means of a Crossbeam 540 system. For analysis of the EBSD data the software OIM v7.2.1 (Orientation Imaging Microscopy) distributed by EDAX company was used. A special challenge in performing EBSD measurements at the material system under discussion is the fact that the two components of the electrode's active material, LiMn2O4 (LMO) and LiMn1/3Co1/3Ni1/3O2 (NMC), feature different crystal structures based on different crystal systems and space groups. Since it is not possible to determine the crystal structure of the material under investigation by the method of EBSD, crystallographic data has to be provided and preset to the software. Although the two unit cells seem to be sufficiently different to allow clear distinction, wrong automatic assignment was frequently observed during the measurements. Measuring-spots within a LMO particle were assigned to the crystal structure of NMC and vice versa. However, the software allows correcting these misassignments by correlating the results of EBSD and EDX measurements. A specific phase identified in an EDX map of the surface under investigation which is characterized by the chemical composition determined by EDX can be manually assigned to a preset crystallographic phase from the EBSD map. The analysis of the orientation relations for various obviously cohering grains containing surprisingly two different colors (suggesting two different orientations) in the inverse pole figure image yielded the same result for several spot checks within different grains. Especially the observed misorientation angle was $90^{\circ}$. This result pointed to the presence of a pseudo-symmetry. The software allows compensating for such pseudo-symmetries by an implemented cleanup procedure. The execution of this procedure corrects the image data straightening out the inverse pole figure image. In addition a single step grain dilation cleanup can help to further smooth the inverse pole figure image especially near the grain boundaries. The resulting image is shown in Figure 1.

The procedure presented in this study allows to perform electron backscatter diffraction measurements at cross sections on thin foils like lithium ion battery cathodes to access the crystallographic microstructure. In the model case described it was possible to resolve the isotrop orientation distribution within NMC agglomerates used in mixed NMC / LMO (1:1) LIB cathodes. The correlation to Li storage and release properties, described at the beginning will be investigated in the next step.

References:

[1] A. J. Wilkinson et al., Materials Today 15 (2012), p. 366.

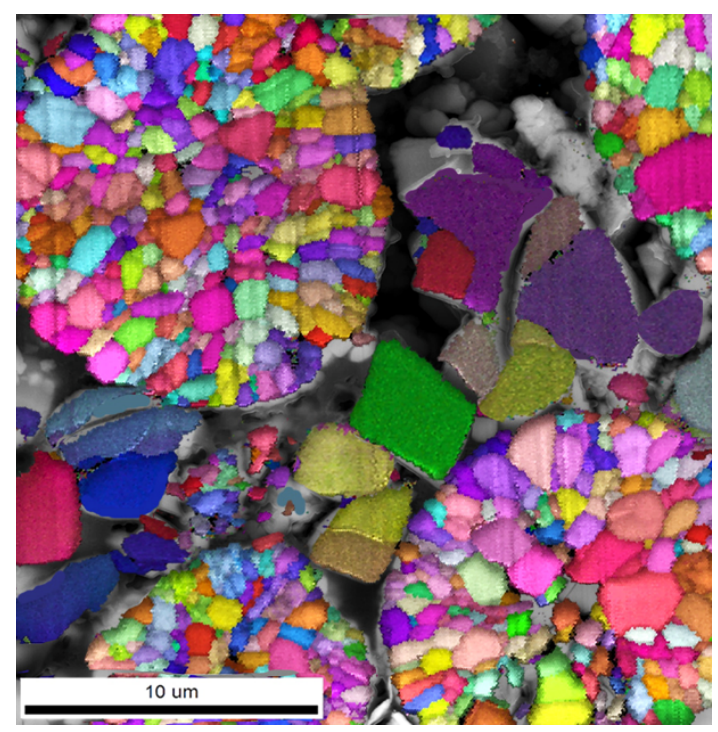

Figure 1. EBSD-analysis for the visualization of crystal orientation and the granulate sub-grain size of blended cathode active material in 18650 lithium ion batteries, Crossbeam 550 SEM . 